\title{
Small UAV Camera Gimbal Stabilization Using Digital Filters and Enhanced Control Algorithms for Aerial Survey and Monitoring
}

\author{
Miroslav LAŠŠÁK $K^{1}$, Katarína DRAGANOVÁ*2, Monika BLIŠŤANOVÁ \\ Gabriel KALAPOS̆4 and Juraj MIKLOS $\check{S}^{5}$
}

Authors' affiliations and addresses:

${ }^{1}$ Flight Management and Control, CoE,

Honeywell International, Inc., Turanka 100, 627

00 Brno, Czech Republic

e-mail: miroslav.lassak@honeywell.com

2, 3,4 Technical University of Košice, Faculty of Aeronautics, Rampová 7, 04121 Košice, Slovakia e-mail : katarina.draganova@tuke.sk

e-mail : monika.blistanova@tuke.sk

e-mail : gabriel.kalapos.1@gmail.com

${ }^{5}$ MiklosTech Contracting Inc., 435 New Brighton Place SE, Calgary, T2Z4W5, Alberta

Canada

e-mail : miklos.juraj.ml@gmail.com

*Correspondence:

Katarína Draganová, Technical University of

Košice, Faculty of Aeronautics, Rampová 7, 041

21 Košice, Slovakia

tel: +421556026153

e-mail: katarina.draganova@tuke.sk

\section{Funding information:}

Slovak Research and Development Agency

Grant Number APVV-18-0248 and APVV-17-

0184

Cultural and Educational Grant Agency

Grant Number KEGA 052TUKE 4/2018,

058TUKE-4/2018

Research Agency

Grant Number 019/2019/1.1.3/OPVaI/DP, ITMS

code 313011 T557.

Acknowledgment:

This work was supported by the APVV-18-0248, APVV-17-0184, KEGA 052TUKE 4/2018,

058TUKE-4/2018 and the VA No.

019/2019/1.1.3/OPVaI/DP with the ITMS code $313011 \mathrm{~T} 557$ projects.

How to cite this article:

Laššák, M., Draganová, K., Blišt'anová, M., Kalapoš, G. and Mikloš, J. (2020). Small UAV Camera Gimbal Stabilization Using Digital Filters and Enhanced Control Algorithms for Aerial Survey and Monitoring. Acta Montanistica Slovaca, Volume 25 (1), 127-137

DOI:

https://doi.org/10.46544/AMS.v25i1.12

\begin{abstract}
Aerial photography, monitoring and survey using small Unmanned Aerial Vehicles (UAVs) is a modern, cheap, simple, helpful and still developing and improving area. For these purposes the ongoing research is focused mainly on the cameras and image processing methods and software. However, as it was confirmed in the article, a stabilized camera gimbal is also very necessary to obtain quality and bright pictures or video records and to allow the operator or the tracking computer to track the camera's line of sight to the point of an interest. Because the camera stabilization is a key factor influencing the quality of the pictures or videos and considering the application on the UAVs performing the flights in the low altitudes and often also in the mountain terrain, the wind conditions, turbulences, wind shears, which can vary in their magnitudes and directions significantly, the convenient stabilization of the camera gimbal can have a significant influence on the quality of the obtained results, which are very important for the creation of the precise $2 \mathrm{D}$ or $3 \mathrm{D}$ models. Furthermore, to increase the UAV payload, it is important to use lightweight solutions. Due to the onboard electronics of small UAVs, regarding the limited memory and computational performance, a small microcontroller including a convenient, simple, and still fast enough control algorithm needs to be designed and implemented. In order to stabilize a camera gimbal, it is needed to design a model of the actuators as well as the gearings, to propose an effective control algorithm and to implement the control algorithms into the on-board microcontrollers. This article deals with the modelling of the actuator, conventional commercial servomotor used for a camera gimbal stabilization and with the design and verification of the improved control algorithm based on the inverse characteristics of the actuator model. Due to the requirement of the high-quality images, where the fast stabilization is needed, a dynamic correction feedback was implemented. And as the gyroscopes are very sensitive to the UAVs vibrations, the vibrations of the camera gimbal were eliminated by the digital low pass filter. The theoretical background was experimentally verified by the geological survey of the stone pits in Sedlice, Vechec and Klatov in the Eastern Slovakia.
\end{abstract}

\section{Keywords}

unmanned aerial vehicle (UAV), camera gimbal, stabilization, monitoring, photogrammetry, mining areas.

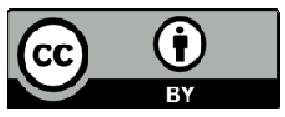

(C) 2020 by the authors. Submitted for possible open access publication under the terms and conditions of the Creative Commons Attribution (CC BY) license (http://creativecommons.org/licenses/by/4.0/). 


\section{Introduction}

Unmanned aerial vehicles (UAVs) have been recently used in various applications. From the monitoring and survey applications in regard to the mining areas (Ren, et al., 2019) as for example, object monitoring, dump, surface subsidence, coal gangue heap, open pit, mining pit or industrial site monitoring can be mentioned. In the terrain surveying and 3D modelling the topographic changes are monitored, earthwork computations are perfomed, dynamic surveys of mine reserves are performed, or soil erosion is estimated. From the ecological and environment monitoring and land damage assessment applications the tasks involving analysis of crop yield decrease is very important, but also surface subsidence, accumulated water, or soil destruction can be monitored (Blistan, et al., 2016). In the case of geological or other disasters, UAVs can be used for pollution monitoring, gas monitoring, coal fire monitoring etc. UAVs also find their applications in the land reclamation and ecological restoration assessment; they can be used, for example, for the monitoring of the vegetation coverage changes, for surveying of the land use or vegetation classification (Ren, et al., 2019). Monitoring of the geological environment of mines is crucial to improving mine environment and reducing and mitigating damage caused by exploitation ( $\mathrm{Li}$, et al., 2015).

For all of the above mentioned applications, aerial photogrammetry, surveillance, and monitoring, including the geological survey, monitoring of Earth resources, of the mining areas, monitoring of the ecological purposes, followed many times with the creation of the 2D and 3D models, the utilization of a camera system is necessary (Blistan, et al., 2019), (Blistan and Kovanic, 2017) and (Lucieer, et al., 2014).

The accurate terrain data are not only the basis of the 3D modeling, but they are also important for the geological risk prediction, due to the fact that the mining exploitation also causes serious damage to the both land and the ecological environment (Ren, et al., 2019), (Xiang, et al., 2018), (Fernández-Lozano, et al., 2018). The framework of UAV applications in the mining activities is presented in the Fig. 1.

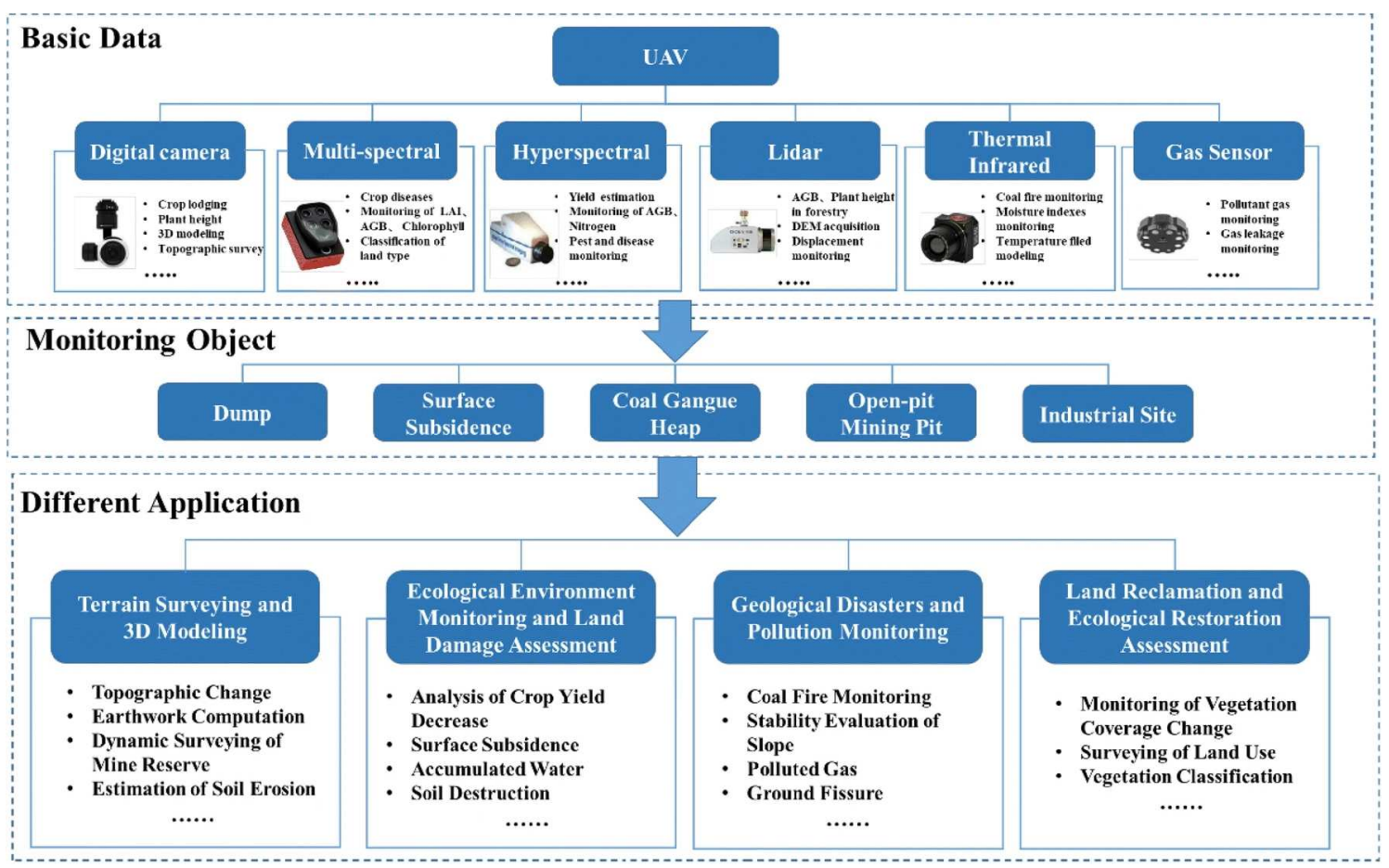

Fig.1. The framework of UAV applications in the mining activities (Ren, et al., 2019 - modified by authors)

The research in the above mentioned areas is mainly focused on the camera design and development and related image or video processing methods and software (Kršák, et al., 2016). The importance of the proper camera gimbal stabilization is often forgotten, although it can have a significant influence on the quality of the resulting camera image or video.

It has to be also mentioned that the category of the small UAVs has in comparison to the conventionally used airborne systems many particularities (Č́ižmár and Jalovecký, 2010), as for example operator demands (Lipovsky, et al., 2019), dimensions, weight, computational demands and last but not least price of the used systems, due to which a new interesting task has arisen: construction of a camera gimbal platform together with the stabilizing and tracking control algorithms fulfilling these requirements. Because without the convenient camera stabilization the UAV itself, the flight manouvers and meteorological conditions, particularly the wind 
and turbulences in the mountain areas and during the flight performed in the low altitudes above ground level, the quality of the images or videos can be decreased significantly.

Recently multiple techniques for the camera gimbal stabilization have been developed. Some of them are based on the PID controllers and on the enhancement of their parameters applying, for example, a feedback loop and steering parameters calculated from quaternion transformation angular velocities received from gyroscopes (Zych, et al., 2015) or evolutionary algorithms such as PSO, GA (Rajesh and Kavitha, 2016) or on the gain scheduling approach with the gimbal model identified in the close loop manner by the PEM algorithm (Golmohammad, et al., 2007) to reduce disturbances and provide better performance and accuracy in the system response due to their effectiveness, simplicity and feasibility. As an alternative approach, the artificial neural networks can provide a more accurate model of the gimbal system based on their non-linear mapping to model the inertial characteristics gyro-stabilized multi-gimbal system (Layshot and Yu, X.-H., 2011). Other approach applicable for the micro and mini UAV category is based on the DC servo motor control and a novel robust control strategy employing the technique of uncertainty and disturbance estimator (UDE) (Kori, Ananda and Chandar, 2016). Other approach uses piezo motors (Karasikov, et al., 2016) instead of the more common servo motors characterized by the higher bandwidth, fast response and direct drive.

For the UAVs of mini and micro categories also gimbals in the role of mechanical video camera stabilizers represent unacceptable payload, thus approaches based on the video stabilization using the computer vision techniques, software image stabilization (Wiriyaprasat and Ruchanurucks, 2015), (Windau. and Itti, 2011), (Pieniạzek, 2003) and video smoothing methodologies (Vazquez and Chang, 2009) have been developed.

Our approach of the camera gimbal stabilization uses a commercial servomotor and the improved control algorithm based on the inverse characteristics of the actuator model with the dynamic correction feedback applying the angular rate signals from the gyroscopes. And thanks to stabilization, better input data in the form of the photos or videos can be achieved, and more precise terrain models can be created.

\section{Materials and Methods}

\section{Unmanned Aerial Vehicles}

Nowadays, on the market, there are available many types of small UAVs. For the monitoring purposes, the mostly preferred are flying vehicles from the category of rotary wings, so-called "multirotors". They differ mainly in the number of rotors (starting from three rotors -"tricopter"). The usage payload is slightly increasing with the number of rotors, the stability is also better when comparing ie. tricopter versus hexacopter with 6 rotors or octocopter comprising of 8 rotors. Flying time of octocopter OktoXL with installed $10000 \mathrm{mAh}$ batteries and an additional payload of 1000 grams reaches still more than 15 minutes, what is enough for monitoring of a small area. An advantage of modular construction of small UAVs is in the possibility of swapping batteries by a spare (fully charged) in several seconds what gives us the ability to continue in the flight mission / survey. In Fig. 2 octocopter predefined for carrying of the camera with the raised landing gear is shown. In the Fig. 2 with the number 1 protective hood is marked, 2 is motor with a 12" propeller, 3 is a rigger, 4 is a landing gear, 5 is a battery holder and finally, as 6 a camera gimbal is labeled.

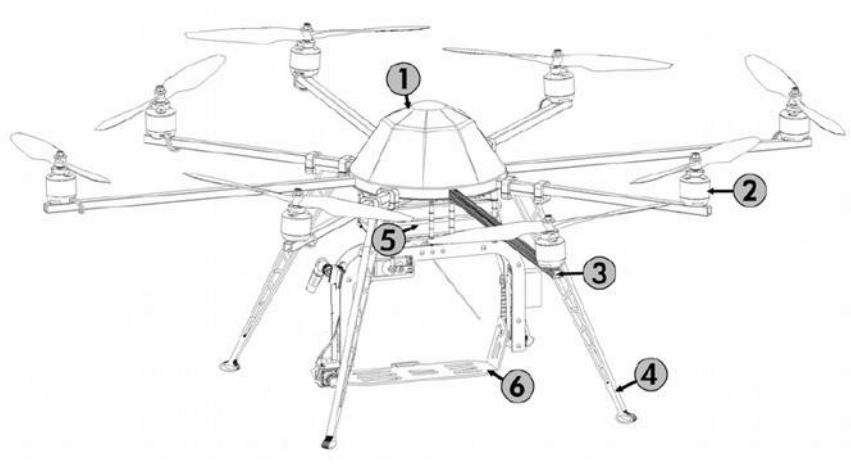

Fig. 2. Octocopter OktoXL (source: https://www.mobilexcopter.com/files/ARFOktoXL-Manual_\%28en\%29.pdf)

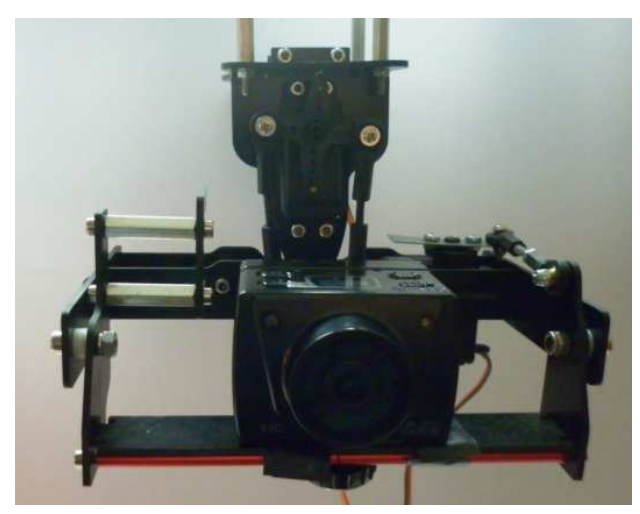

Fig.3. Camera gimbal with the GoPro camera

\section{Camera Gimbal}

Camera gimbals allow us to rotate the camera around each axis, so it is possible to track an object of interest and to have it still in the line of sight (Lozano, 2010). There are many types of camera gimbals used around the world. After the precise analyses of their parameters, cost, weight, and our requirements, the camera gimbal with two degrees of freedom was chosen (Fig. 3). In order to reach the highest precision in the positioning and 
smooth movement, the servomotors with the high number of steps have been used (resulting in the angle resolution less than $0.1^{\circ}$ ).

Considering the multi rotor application, the gimbal will be used to compensate the roll and pitch angles. If the heading needs to be changed, a multi rotor vehicle can rotate around its $z$ axis, meaning that the third gimbal axis is not necessary. As a camera, the high-quality GoPro camera MagiCam SD21 was chosen. This camera is predefined for such applications, as it can make excellent and sharp images even if small vibrations occur.

To give the ground station operator the ability to see online pictures from the flying UAV, the vehicle was equipped with the wireless video transmitter TS 351 (operating at the $5.8 \mathrm{GHz}$ frequency), and in the ground station, the receiver RC 305 was used.

\section{Onboard Sensorics}

To determine the UAV position and the camera gimbal position in the real time, the camera gimbal platform was equipped with the IMU 9 DOF stick (inertial measurement unit with 9 degrees of freedom), the 66 channels GPS receiver Locosys LS20031 and the barometric pressure sensor BMP 102, the data of which were processed by the mbed microcontroller. The used inertial measurement unit includes a 3 axes accelerometer (providing linear acceleration data), a 3 axes gyroscope (providing angular rate data) and a 3 axes magnetomer (needed for the heading computation). All data were onboard stored to a micro SD card. To obtain a better position determination and positioning precision and to eliminate the noise and errors of the used sensors, the complementary filter was used for the INS and GPS data fusion.

\section{Servomotor Simulation Model}

As a servo motor, we used the high-quality Power HD 1209TH servomotor (with the titanium gearings) that allows us to rotate the camera gimbal in the range from $-30^{\circ}$ to $+30^{\circ}$. It's dimensions are $40.3 \mathrm{~mm} \mathrm{x} 20.2 \mathrm{~mm} \mathrm{x}$ $37.2 \mathrm{~mm}$, its weight is just 57 grams, and the maximum angular rate is up to the $750 \%$ s. As it is well known, a servomotor is a complex and nonlinear electromechanical system. We didn't study servomotor's internal construction and gearing, but we modeled it as a "black box" only according to the servomotor's transition characteristics measured using a special workstation. The servomotor was installed on a workstation connected to the external rotational angle sensor (rotational potentiometer), which output data were sampled using the mbed NXP LPC1768 microcontroller at the $10 \mathrm{kHz}$ rate. As soon as the primary measured data were analyzed, the transport delay of $20 \mathrm{~ms}$ was determined. At this moment, we were aware of the difficulties that we will face during the control algorithm design process (the instability when the regulator is fast, long regulation time). After additional precise analyses were completed, the simulation model was designed and also simplified using the Mason's gain formula. The simplified simulation model of the Power HD 1209TH Servomotor in the Matlab Simulink is shown in the Fig. 4.

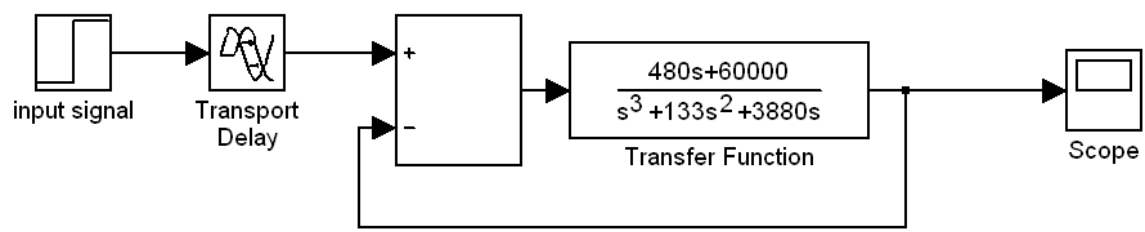

Fig. 4. Simplified simulation scheme of the POWER HD 1209 TH servomotor

The obtained simulation results were compared with the real measured data from the servomotor, and the correctness of the proposed model was confirmed. As it is clear from the Fig. 5, where the results from the step responses are displayed, the simulation model is precise enough.

For the quantitative evaluation of the proposed model, the following statistical values were calculated: maximum absolute average error (MAAE), mean absolute average error (MAE), mean absolute percentage error (MAPE) and quadratic integral error (QIE):

MAAE $=2.916^{\circ}$

$\mathrm{MAE}=1.284^{\circ}$

MAPE $=0.0469 \%$

$\mathrm{QIE}=9.1406 .10^{-4}$

After the successful servomotor simulation model design, the complex model of the whole gimbal was developed, considering the camera gimbal's gearing in the roll and pitch axis, too. 


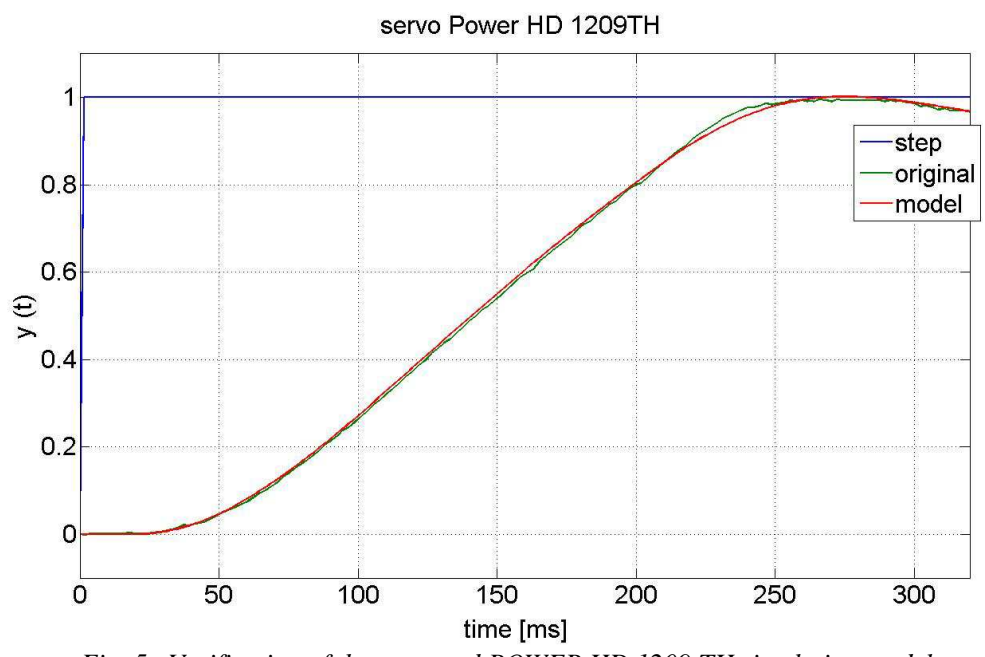

Fig. 5. Verification of the proposed POWER HD 1209 TH simulation model.

\section{Results and Discussion}

As it was mentioned earlier, used servomotors have the transport delay that makes the control algorithm design quite difficult. There are many robust control algorithms primary designed for the systems with the transport delay, but usually, they are slow and have high computational demands. Considering the onboard microcontroller that will be used for the camera gimbal stabilization, our goal was to use as simple algorithm as possible, obviously with the satisfactory results. Firstly we decided for an open-loop control algorithm based on an inverse function of the mathematical model. For the transport delay elimination, the corrective element was used. Based on the theory (Bakshi and Bakshi, 2009), the dynamic parameters of the controlled object can be modified via a closed-loop corrective element. The main principle of the corrective element is to add a closed loop, which needs to guarantee the stability and quality, and to add an additional signal, initially not used in the control algorithm, that improves the controlling process (Madarász, et al., 2009). In our application, the dynamic correction was performed; the angular rate signals from gyroscopes were added to the actuating signal $u_{r}$ computed by the inverse model controller (IMC) as it is illustrated in the Fig. 6.

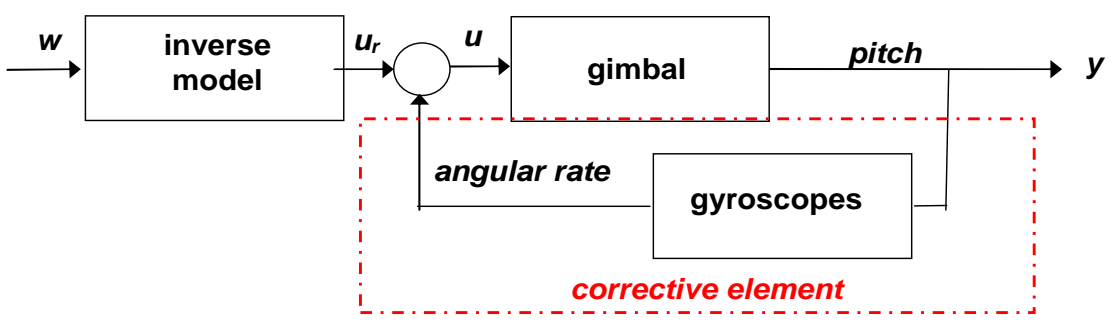

Fig. 6. Block scheme of the inverse model control loop with the dynamic correction.

From the control algorithms theory, for the stability purposes, only the middle frequency asymptote is important (it has to have the " -1 " slope) (Bakshi and Bakshi, 2009). The gyroscopes, as the angular rate sensors, can be considered as a source of a high-frequency signal - as they sense fast changes in the direction. For the reduction of the possible high frequency (disturbing) noise (noise dynamics is considerably higher than the servomotors dynamics), the low pass filter was applied for the gyroscopes' data. The low pass filter transfer function is:

$$
F(s)=\frac{1}{T_{1} s+1}
$$

where $T_{1}$ is the filter time constant.

As the control algorithm was implemented in the mbed microcontroller, the necessity of the digital filtering algorithms creation and implementation in the microcontroller arose. Due to the high demands on the dynamics and the lowest possible delay, the IIR (Infinite Impulse Response) filter was chosen (Vaispacher, Andoga, Bréda, Adamčík, 2015). 


\section{IIR Filter}

The low pass filter, needed for the angular rate data filtering, with the window size of 2 samples, was designed based on (Steven, 1997). The advantage of the IIR filters in comparison with the FIR (Finite Impulse Response) filters lies in the better filtering ability and the lower time delay. The main disadvantage of IIR filters is the possibility to be unstable.

IIR filter design approach: Consider the data that has to be filtered as an input signal for the filter, in our application, the angular rate, $x_{I I R}$. The output data from the filter are marked as $y_{I I R}$. In the discrete-time domain, the input data is one column vector (with the length of $L$ ), with samples numbered as $1,2,3, \ldots L$. Simple, the IIR filter principle is based on the iterative algorithm. The output signal is computed from the actual input data $x(n)$, previous input data $(x(n-1), x(n-2), \ldots$.$) , and also the previous output data (y(n-1), y(n-2), \ldots$.$) .$

In general, the formula for such IIR filter is known as a recursive equation:

$$
\begin{aligned}
& y_{I I R}(k)=a_{0} x_{I I R}(k)+a_{1} x_{I I R}(k-1)+a_{2} x_{I I R}(k-2)+ \\
& +a_{3} x_{I I R}(k-3)+\cdots+b_{1} y_{I I R}(k-1)+b_{2} y_{I I R}(k-2)+b_{3} y_{I I R}(k-3)+\cdots
\end{aligned}
$$

where $y_{I I R}$ is the output filter data, $x_{I I R}$ are input filter data (raw data), $k$ represents the number of samples, $a_{0} \ldots a_{3}$ are coefficients, which the input filter data are multiplied by and $b_{1} \ldots b_{3}$ are coefficients, which the output filter data are multiplied by.

The design of the used low pass IIR filter can be explained on a simple example, where one output sample and two input samples will be used. For a sample frequency $f_{s}$ and a cut-off frequency $f_{c}$ the following relationship can be written:

$$
z=e^{-2 \Pi f_{c} / f s}
$$

where the criterion $f_{\mathrm{c}} / f_{\mathrm{s}}$ falls into the interval $(0,0.5)$

For the low pass filter, the coefficients can be computed as:

$$
a_{0}=1-z ; b_{1}=z
$$

In our application, the sampling frequency $f_{s}=100 \mathrm{~Hz}$ was used. Based on the experiments and analyses of the gimbal dynamics, the cut-off frequency was set to $f_{c}=1 / 6 \mathrm{rad} \cdot \mathrm{s}^{-1} \sim 1 \mathrm{~Hz}$. When the input signal is at the frequency of $f=20 \mathrm{~Hz}\left(f / f_{\mathrm{s}}=0.2\right)$, the attenuation of $-9 \mathrm{~dB}$ occurs.

The filtering ability verification of the designed IIR filter applied to the real measured angular rate data can be seen in the Fig. 7.

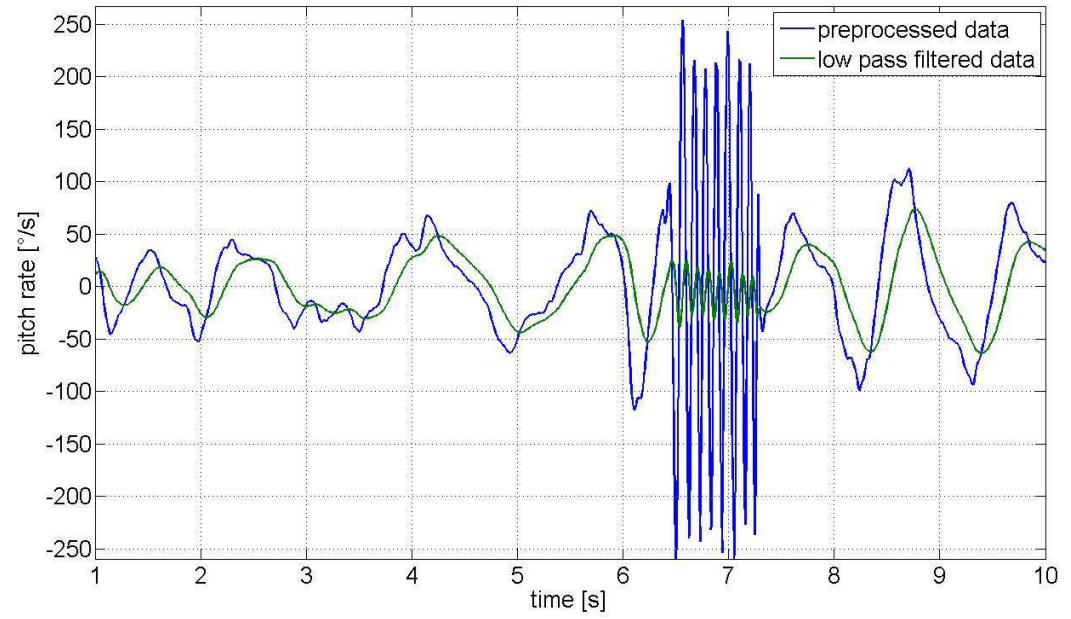

Fig. 7. IIR low pass filter verification (angular rate data as the input)

As it can be seen from the Fig. 7, the IIR filter filters out the high frequency „,noise“, vibrations that occurred at the time between 6-7 seconds. When the multi-rotor realized a smooth movement, the IIR filter passed the input data without any attenuation (Vaispacher, Bréda, Madarász, 2015). 


\section{Inverse Model Controller}

Based on the aforementioned analyses, the inverse model controller with the dynamic correction was designed and simulated with the gimbal simulation model in the control loop (see Fig. 8).

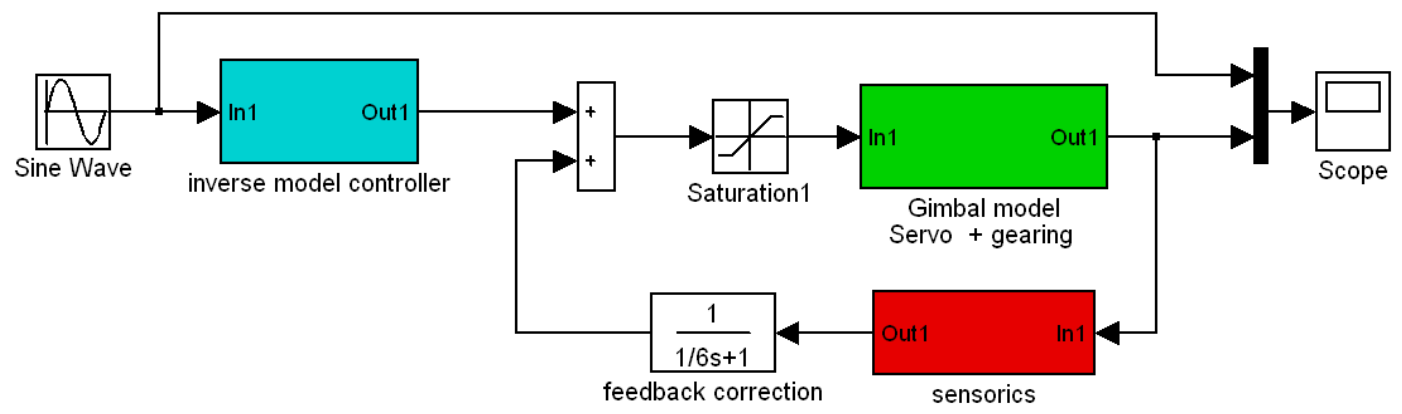

Fig. 8. The simulation scheme of the inverse model controller with the dynamic correction

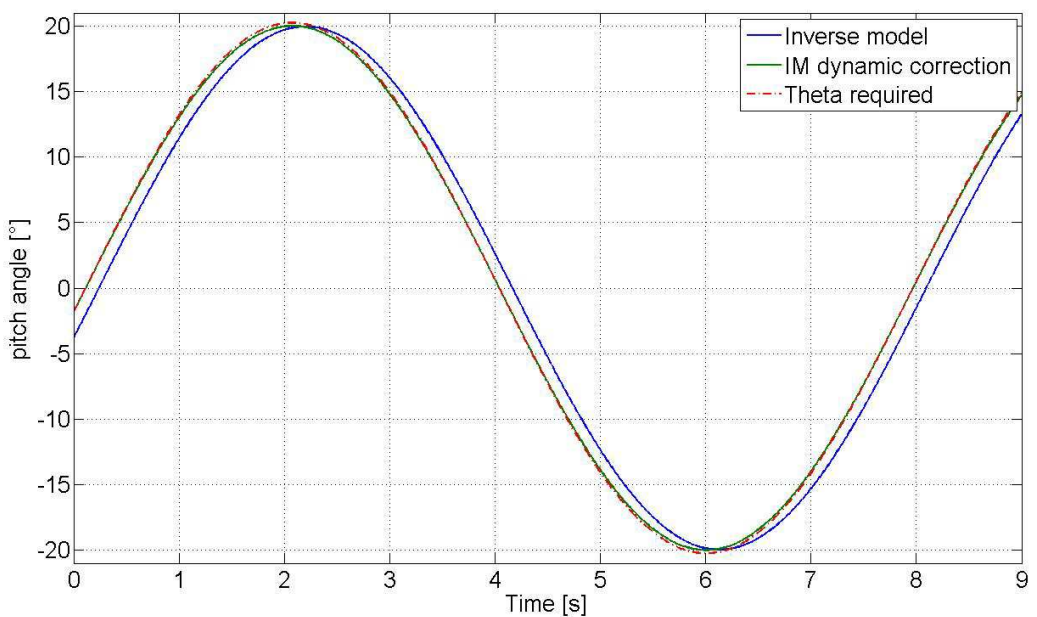

Fig. 9. The inverse model controller simulation verification

Running plenty of simulations of the designed camera gimbal control algorithms with a variety of input signals, the maximum time delay between the command and measured gimbal output of $13 \mathrm{~ms}$ (without the dynamic correction), and only $1 \mathrm{~ms}$ with the dynamic correction was determined. Running the simulation with the steps input signal, the maximum steady-state error of $0.04^{\circ}$ in the pitch and $0.06^{\circ}$ in the roll angle was determined.

The correct inverse model controller behaviour was confirmed by the Fig. 9. A significant improvement in the performance of the inverse model controller with the dynamic feedback correction against the conventional inverse model controller, especially in the regulation time, can be seen from the the Fig. 9. During the inverse model controller simulation, the sinusoidal signal as the input signal (simulating continuously changing pitch angle with the amplitude of $20^{\circ}$ ) was used. For the regulator verification, only the sine wave signal is presented, as the sine wave signals represent the possible movement of the UAV during manoeuver, climbing, descending, rotation around $\mathrm{z}$-axes, or movement due to the wind. However, better results in the regulation time, due to angular rate signals used by the control law, were reached for all input signals (step, ramp, etc.).

Tab. 1. Statistical errors for the controller verification

\begin{tabular}{ccc} 
& Tab. 1. Statistical errors for the controller verification \\
\hline & IMC without the dynamic correction & IMC with the dynamic correction \\
\hline$M A A E\left[^{\circ}\right]$ & 2.0497 & 0.2523 \\
\hline$M A E\left[^{\circ}\right]$ & 1.3916 & 0.1510 \\
\hline$M A P E[\%]$ & 0.6611 & 0.0180 \\
\hline IIE $[-]$ & 2.3200 & 0.0280 \\
\hline
\end{tabular}

From the Tab. 1 with overviewed statistical errors can be seen that in the case of the inverse model controller with the dynamic correction, the decrease in the achieved statistical errors, which means an increase in the quality of the control process, is obvious. 


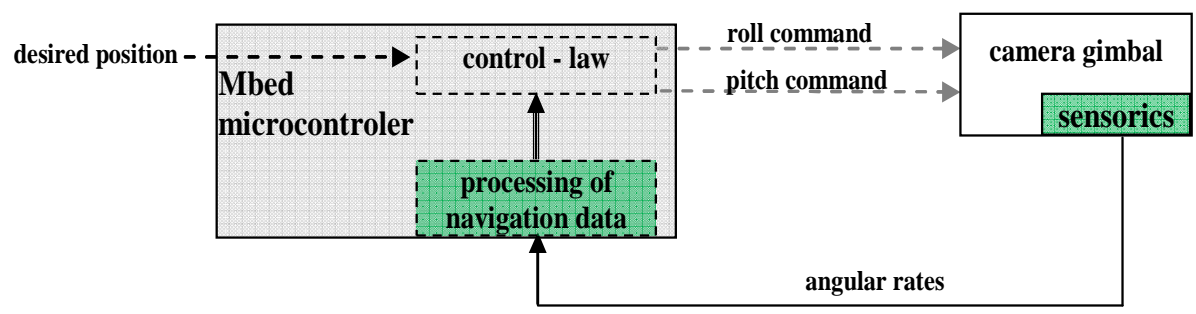

Fig. 10. Hardware implementation

As the proposed inverse model controller was successfully verified by the simulation, it was implemented into the onboard mbed microcontroller (Fig. 10.) and successfully tested on the real camera gimbal for the UAV with the satisfactory results.

\section{Geological Monitoring and Photogrammetry}

When flying with the small and lightweight UAVs, even a small gust of wind can significantly affect their flight, and the UAV has to compensate these disturbances continuously via changing the pitch and roll (or yaw angle) and position itself. To obtain high quality images and videos, the camera gimbal is compensating for the UAV movements (position angles) as well as tracking the object of an interest.

The proposed above-mentioned methodology was also tested experimentally. A geological survey was performed using the UAV - Mikrokopter Okto XL in cooperation with the Future Fly company that is an owner of the Aerial Work Operator Certificate No. SK/096, in the stone, pits Sedlice shown in the Fig. 11., Vechec (Fig. 12) and Klatov (Fig. 13) in the Eastern Slovakia. Based on the dimensions of the monitored area, photos were taken from the height of approximately 500 meters. The camera gimbal stabilization and precise positioning are extremely important as just small vibrations caused by the UAV's rotors, the noise of inertial sensors used for the stabilization, or noise of positioning servo motors have a significant influence on the image quality. Taken into account the $500 \mathrm{~m}$ height, vibrations in the camera line of sight with the amplitude of just 0.1 degrees will result in the error of \pm 0.9 meters and accordingly blurred photos.

Image shown in the Fig. 13 was taken in the Klatov area from the height $150 \mathrm{~m}$ above ground level, and subsequently, they were processed into the 3D model using the Agisoft PhotoScan software, as can be seen in the Fig. 14. In this area, the first set of testing pictures were taken without the enhanced stabilization of the camera gimbal (Fig. 15 and Fig. 16), and these pictures were then compared to the images taken with the active camera gimbal stabilization (Fig. 17 and Fig. 18). As can be clearly seen from the pictures, the proposed and tested stabilization of the camera gimbal lead to the higher sharpness of the image and minimal noise.

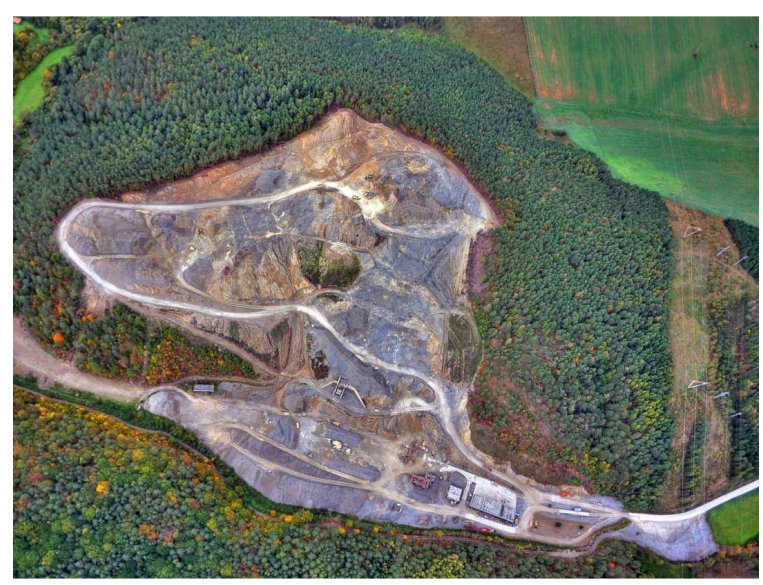

Fig. 11. Aerial survey of the open pit mine Sedlice.

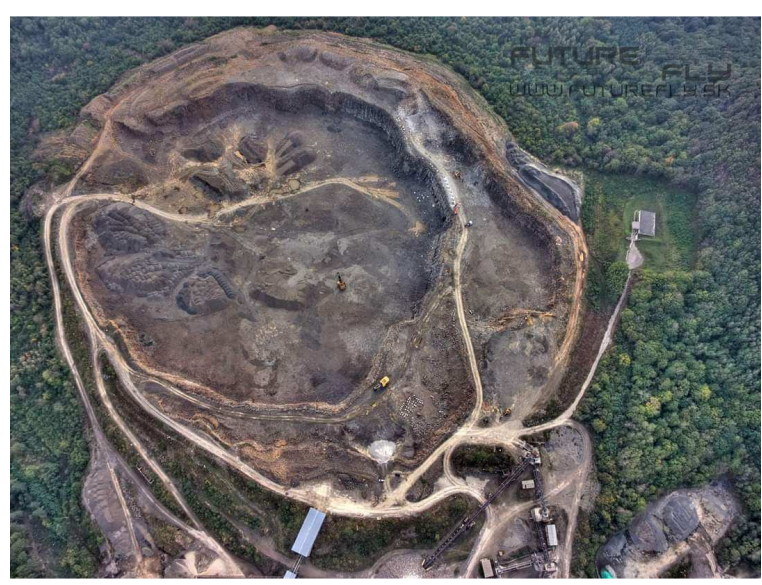

Fig. 12. Aerial survey of the open pit mine Vechec. 


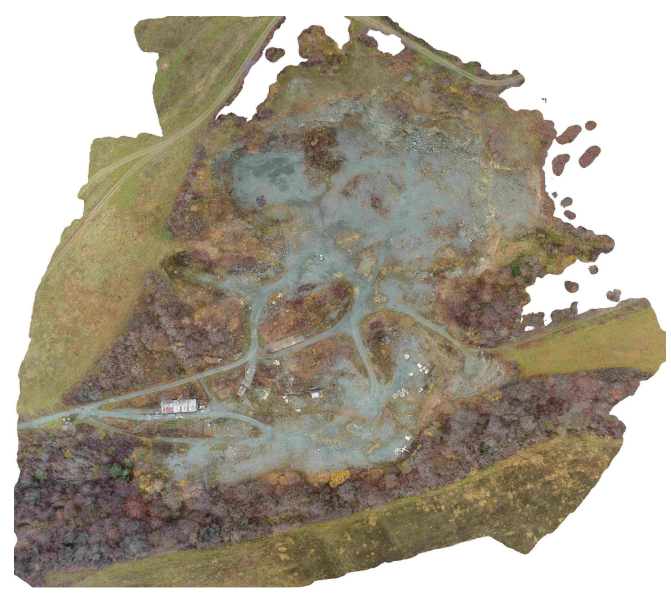

Fig. 13. Aerial survey of the open pit mine Klatov.

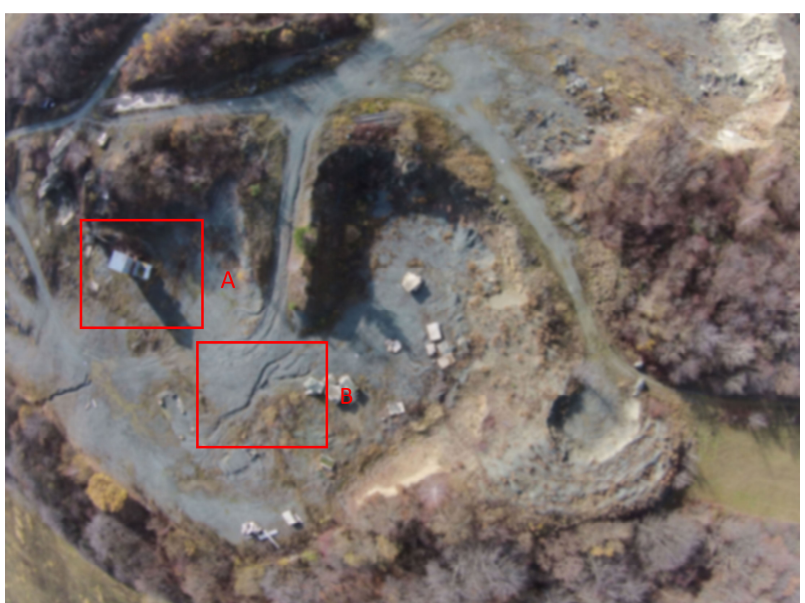

Fig. 15. Photos taken without the enhanced camera gimbal stabilization ( $A$ - building, $B$ - grooved terrain).

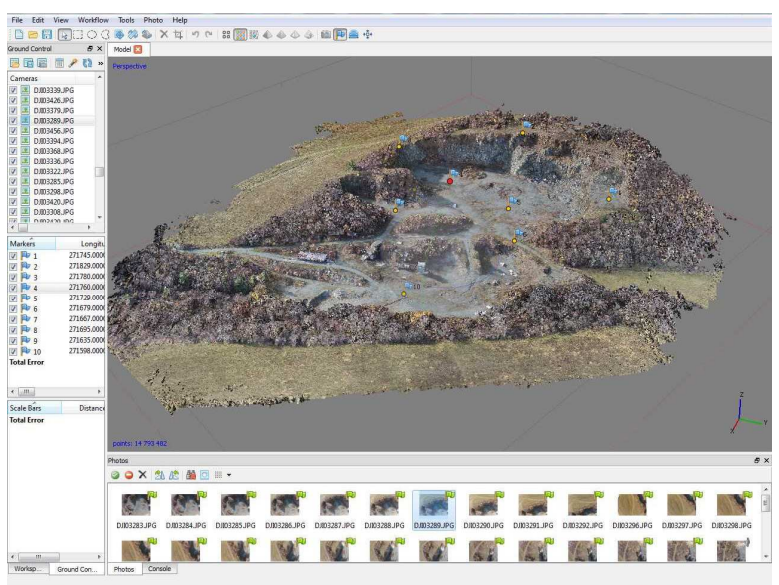

Fig. 14. Image processing using the PhotoScan software.

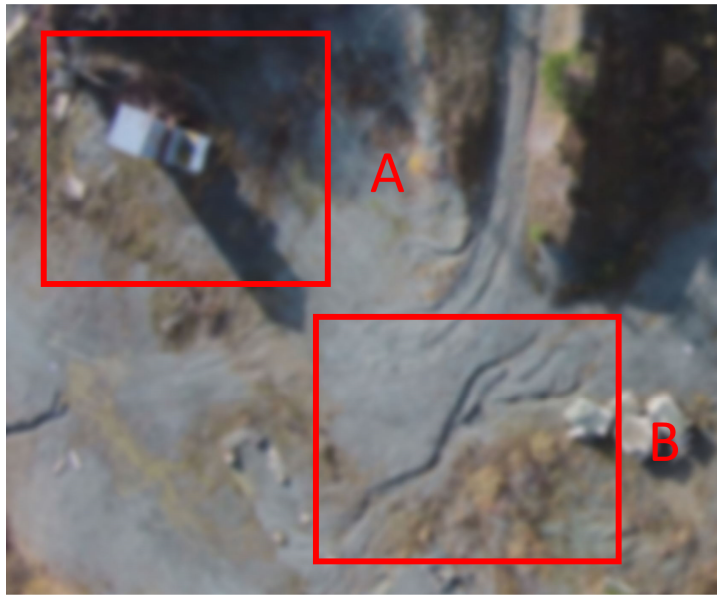

Fig. 16. Detail of the objects of interest without the enhanced camera gimbal ( $A$ - building, $B$ - grooved terrain).

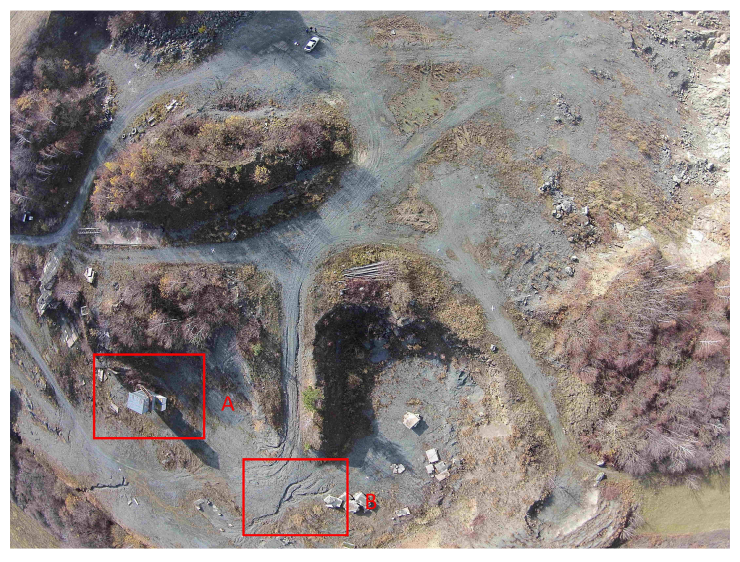

Fig. 17. Photos taken with the enhanced camera gimbal stabilization ( $A$ - building, $B$ - grooved terrain).

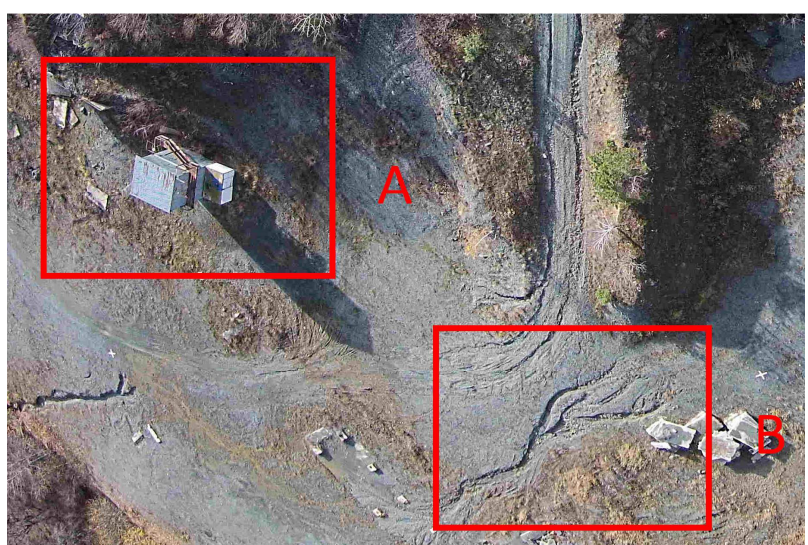

Fig. 18. Detail of the objects of interest without the enhanced camera gimbal ( $A$ - building, $B$ - grooved terrain).

In addition to the obviously seen functionality of the proposed camera gimbal stabilization from the photos shown in Fig. 15 - Fig. 18., the correctness of the theoretical principles together with their implementation and practical realization on the UAV was also proved by the fact that the resulting error of the image resolution per pixel was reduced in compliance with the theoretical assumptions almost ten times. 


\section{Conclusion}

Aerial survey and monitoring using small Unmanned Aerial Vehicles (UAVs) are modern, cheap and still rapidly developing and improving the area, which can be used for a wide band of various applications, such for example aerial photography, photogrammetry, surveillance, and monitoring, including geological survey, monitoring of Earth resources, of the mining areas, monitoring of the ecological purposes, natural disasters, etc., for which utilization of a camera system is required. For these purposes, a stabilized camera gimbal is necessary to obtain quality and bright pictures and to allow the operator or the tracking computer to track the camera's line of sight to the point of interest. Considering these applications, a very challenging task is to design a properly stabilized camera gimbal, which can compensate the vibrations caused by the UAV construction and operation itself, which is able to whitstand also the performed maneuvers and also which is resistant to the wind conditions, that can cause significant problems with the stabilization, especially considering the UAV utilization in the low altitudes and in the mountain terrain. In order to stabilize the camera gimbal, it is needed to design a model of the actuators as well as the gearings, to propose an effective control algorithm and to implement the control algorithms into the onboard microcontrollers. As the payload of the used vehicle is important, the small and lightweight single cheap microcontrollers have to be used. Due to the limited memory and computational power of the small microcontroller, a convenient, simple, and still fast enough control algorithm needs to be designed and implemented. This article deals with the modeling of the actuator, conventional commercial servomotor, used for the camera gimbal stabilization, and with the design and verification of the improved control algorithm based on the inverse characteristics of the actuator model. As for the high-quality images, the fast stabilization is needed, dynamic correction feedback based on the angular rates signals from the gyroscopes to the computed command was added. Considering the fact that gyroscopes measure fast rotational movements, they are very sensitive to the UAVs rotor vibrations, are noisy, and so the elimination of the undesirable vibrations of the camera gimbal applying the digital low pass filter for the angular rates signal is needed. As the microcontroller is working in the discrete-time domain, a discrete digital filter was designed.

UAVs nowadays are inexpensive and have wide applicability also in the mining applications. The correctness of the proper inertial sensors data fusion, data filtering, and fast camera gimbal stabilization was therefore proven experimentally in the geological survey of the stone pits, using the UAV of the octocopter configuration, where bright pictures were obtained. As UAVs are able to scan the terrain rapidly and cheaply, also other experimental flights were performed in the stone pit area. In this case, the images obtained from the UAV during the test flight without the enhanced camera gimbal stabilization were compared to the photos taken with the proposed camera gimbal stabilization. The results of the further image processing confirmed the theory and approximately ten times better results were achieved.

The further research will be focused mainly on the optimization of the control algorithm also in connection to the fast-developing onboard sensors that can be used for the camera gimbal stabilization, which will significantly improve the quality of the images, which is determining for the further postprocessing in software used in the photogrammetry, for the texture information in the topography and mainly for the creation of the 3D terrain models in the areas, where the changes and variations are monitored and evaluated.

\section{References}

Bakshi, U.A, Bakshi, V.U. (2009). Automatic Control Systems. Technical Publications.

Blistan, P.; Kovanič, L.; Zelizňaková, V.; Palková, J. (2016) Using UAV photogrammetry to document rock outcrops. Acta Montanistica Slovaca, 21 (2).

Blistan, P., Kovanic, L. (2017). Verification of usability of low-cost UAV photogrammetry in comparison with close-range photogrammetry in the context of documentation of Earth's surface. Geographic Information Systems Conference and Exhibition (GIS ODYSSEY 2017).

Blistan, P, Kovanic, L., Patera, M., Hurcik, T. (2019). Evaluation quality parameters of DEM generated with low-cost UAV photogrammetry and Structure-from-Motion (SfM) approach for topographic surveying of small areas. Acta Montanistica Slovaca, 24 (3).

Čižmár, J., Jalovecký, R. (2010). Design of an Inertial Reference Unit with the mixed gravitational and magnetic correction of gyroscopic drift. Transport Means - Proceedings of the International Conference (2010).

Fernández-Lozano, J., González-Díez, A., Gutiérrez-Alonso, G., Carrasco, R.M., Pedraza, J., García-Talegón, J., Alonso-Gavilán, G., Remondo, J., Bonachea, J., Morellón, M. (2018). New Perspectives for UAV-Based Modelling the Roman Gold Mining Infrastructure in NW Spain. Minerals , 8 (11).

Golmohammad, H., Homaei, A., Saadat, S. (2007). Design and implimentation of an adaptive PI controller for a two axis gimbal system. IFAC Proceedings Volumes (IFAC-PapersOnline), 17, Part 1. 
Karasikov, N., Peled, G., Yasinov, R., Yetkariov, R. (2016). Piezo-based miniature high resolution stabilized gimbal. Proceedings of SPIE - The International Society for Optical Engineering 9828. DOI: $10.1117 / 12.2225623$.

Kori, A.S., Ananda, C.M., Chandar, T.S. (2016). Robust control of single axis gimbal platform for micro air vehicles based on uncertainty and disturbance estimation. Proceedings of 2016 7th International Conference on Mechanical and Aerospace Engineering. DOI: 10.1109/ICMAE.2016.7549588.

Kršák, B., Blišt’an, P., Pauliková, A., Puškárová, P., Kovanič, L., Palková, J., Zelizňaková, V (2016). Use of low-cost UAV photogrammetry to analyze the accuracy of a digital elevation model in a case study. Measurement, 91.

Layshot, N., Yu, X.-H. (2011). Modeling of a gyro-stabilized helicopter camera system using artificial neural networks. 2011 IEEE International Conference on Information and Automation, ICIA. DOI: 10.1109/ICINFA.2011.5949035.

Li, Y., Zhao, H. and Fan, J., (2015). Application of remote sensing technology in mine environment monitoring. MATEC Web of Conferences 22, DOI: 10.1051/matecconf/20152204008

Lipovsky, P., Szoke, Z., Moucha, V., Jurc, R., Novotnak, J. (2019). Data acquisition system for uav autopilot and operator evaluation. MOSATT 2019 - Modern Safety Technologies in Transportation International Scientific Conference, Proceedings. DOI: 10.1109/MOSATT48908.2019.8944105

Lozano, R. (2010). Unmanned aerial vehicles : Embedded control. Wiley.

Lucieer, A., Jong, S.M.D., Turner, D. (2014). Mapping landslide displacements using Structure from Motion (SfM) and image correlation of multi-temporal UAV photography. Progress in Physical Geography, 38 (1).

Madarász, L., Andoga, R., Főző, L., Lazar, T. (2009) Situational control, modeling and diagnostics of large scale systems. Studies in Computational Intelligence, 243. DOI: 10.1007/978-3-642-03737-5_11.

Pieniązek, J. (2003). Software-based camera stabilisation on unmanned aircraft. Aircraft Engineering and Aerospace Technology, 75 (6). DOI: 10.1108/00022660310503048.

Rajesh, R.J., Kavitha, P. (2016). Camera gimbal stabilization using conventional PID controller and evolutionary algorithms. IEEE International Conference on Computer Communication and Control, IC4 2015. DOI: 10.1109/IC4.2015.7375580.

Ren, H., Zhao, Y., Xiao, W., Hu, Z. (2019). A review of UAV monitoring in mining areas: current status and future perspectives. International Journal of Coal Science \& Technology, 6. DOI :10.1007/s40789-01900264-5.

Steven, W. S. (1997). The Scientist and Engineer's Guide to Digital Signal Processing Recursive Filters. 2nd edition. California Technical Publishing.

Vaispacher, T., Andoga, R., Bréda, R., Adamčík, F. (2015). Application of linearized Kalman filter in integration of navigation systems. CINTI 2015 - 16th IEEE International Symposium on Computational Intelligence and Informatics, Proceedings. DOI: 10.1109/CINTI.2015.7382898.

Vaispacher, T., Bréda, R., Madarász, L. (2015). Integration architectures of navigation systems for unmanned vehicles. SAMI 2015 - IEEE 13th International Symposium on Applied Machine Intelligence and Informatics, Proceedings. DOI: 10.1109/SAMI.2015.7061854.

Vazquez, M., Chang, C. (2009). Real-time video smoothing for small RC helicopters. Conference Proceedings IEEE International Conference on Systems, Man and Cybernetics. DOI: 10.1109/ICSMC.2009.5346659.

Windau, J., Itti, L. (2011). Multilayer real-time video image stabilization. IEEE International Conference on Intelligent Robots and Systems.DOI: 10.1109/IROS.2011.6048344.

Wiriyaprasat, K., Ruchanurucks, M. (2015). Realtime VDO stabilizer for small UAVs using a modified homography method. Proceedings 2015 International Conference on Science and Technology, TICST. DOI: 10.1109/TICST.2015.7369337.

Zych, C., Wrońska-Zych, A., Dudczyk, J., Kawalec, A. (2015). A correction in feedback loop applied to twoaxis gimbal stabilization. Bulletin of the Polish Academy of Sciences: Technical Sciences, 63 (1). DOI: 10.1515/bpasts-2015-0025.

Xiang, J., Chen, JP., Sofia, G., Tian, Y., Tarolli, P. (2018). Open-pit mine geomorphic changes analysis using multi-temporal UAV survey. Environmental Earth Sciences, 77 (6). 